\title{
Mesh and plane selection: a summary of options and outcomes
}

\author{
Yewande Alimi', Chamilka Merle', Michael Sosin², Marielle Mahan³, Parag Bhanot ${ }^{1}$ \\ 'Department of Surgery, Medstar Georgetown University Hospital, Washington, DC 20007, USA. \\ ${ }^{2}$ NYU Langone Health, The Hansjörg Wyss Department of Plastic Surgery, New York, NY 10016, USA. \\ ${ }^{3}$ Department of Medicine, Medstar Washington Hospital Center, Washington, DC 20010, USA.
}

Correspondence to: Dr. Parag Bhanot, Department of Surgery, Medstar Georgetown University Hospital, 3800 Reservoir Rd NW Washington, DC 20007, USA.E-mail: PXB129@gunet.georgetown.edu

How to cite this article: Alimi Y, Merle C, Sosin M, Mahan M, Bhanot P. Mesh and plane selection: a summary of options and outcomes. Plast Aesthet Res 2020;7:5. http://dx.doi.org/10.20517/2347-9264.2019.39

Received: 15 Oct 2019 First Decision: 9 Dec 2019 Revised: 1 Jan 2020 Accepted: 15 Jan 2020 Published: 20 Feb 2020

Science Editor: Sahil Kuldip Kapur Copy Editor: Jing-Wen Zhang Production Editor: Tian Zhang

\begin{abstract}
Abdominal wall reconstruction is a relevant and important topic not only in plastic and reconstructive surgery, but in the practice of general surgeons. The ideal anatomic location for mesh placement during the repair of ventral hernias has been debated; however, the most common anatomic locations include onlay, inlay, sublay-retromuscular, sublaypreperitoneal, and sublay-intraperitoneal techniques, as defined by the European Hernia Society. Additionally, the availability of numerous synthetic and biologic meshes on the market provides for several options for the practicing surgeon. In this review, we provide a summary of the available literature of both the ideal mesh plane and the appropriate opportunities to use both synthetic and biologic meshes.
\end{abstract}

Keywords: Ventral hernia repair, mesh, underlay, onlay, inlay, sublay, synthetic mesh, biologic mesh

\section{INTRODUCTION}

Defects in the anterior abdominal wall with a resulting ill-defined bulge are both aesthetically displeasing and are associated with musculoskeletal and gastrointestinal dysfunction. The field of abdominal wall reconstruction is a complex topic that comprises a large practice approached by both plastic and reconstructive surgeons and general surgeons. Over one-quarter of all individuals have or will develop a hernia in their lifetime ${ }^{[1]}$. Although the true incidence of incisional hernias is difficult to ascertain,

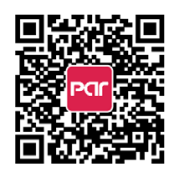




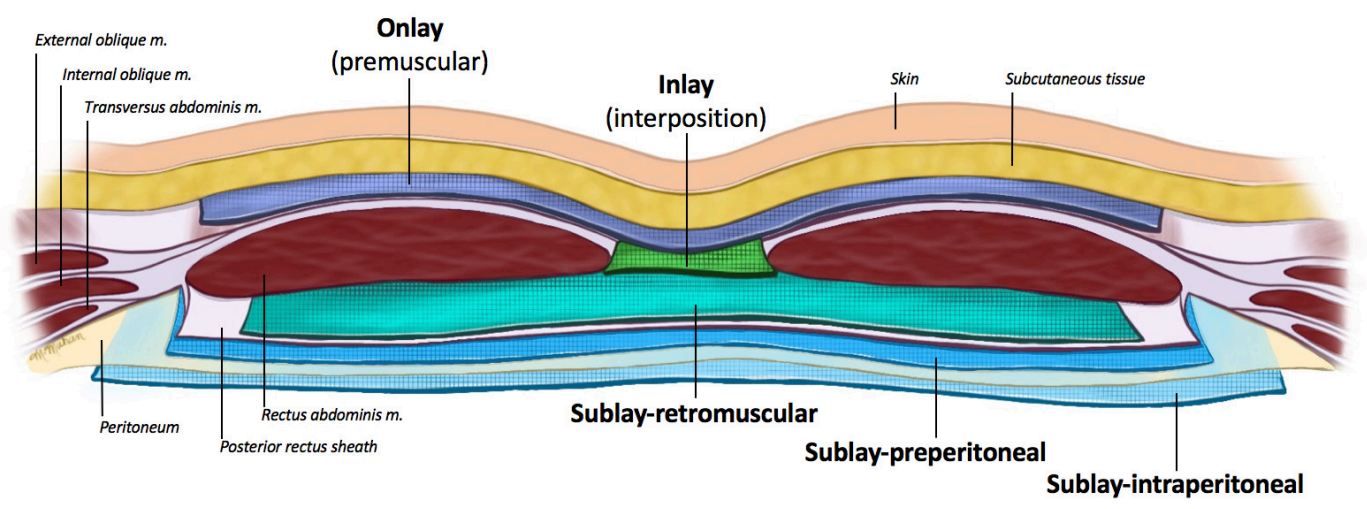

Figure 1. Abdominal wall demonstrating mesh planes

approximately 348,000 hernia repairs were performed in the United States in $2006^{[2]}$. Ventral hernias are an encapsulating term referring to anterior abdominal wall hernias that include the following: epigastric, umbilical, spigelian, parastomal, and most incisional hernias. In the United States alone, over 3.4 billion dollars are spent on the management of hernias ${ }^{[1]}$. The standard approach to ventral hernia repair and the realm of abdominal wall reconstruction is that of repair of the fascial defect with reinforcement of the abdominal wall with mesh. There are many facets to the completion of a ventral hernia repair, including approach to repair, mesh type selection, and mesh plane selection. The approach to the repair can be completed open or through minimally invasive techniques. Presently, minimally invasive techniques utilize both laparoscopic and/or robotic platforms. The selection of the mesh can be that of a prosthetic or biologic variety. Finally, the location in which the selected mesh is placed is crucial to the integrity of the repair. The focus of this review is the latter of these three facets: the choice of mesh and the anatomic location of mesh.

\section{MESH LOCATION}

The ideal anatomic location for mesh placement during the repair of ventral hernias or abdominal wall reconstruction has been debated; however, the most common anatomic locations include: onlay, inlay, sublay-retromuscular, sublay-preperitoneal, and sublay-intraperitoneal [Figure 1]. Numerous singleinstitution studies, reviews, and meta-analysis have been completed on this topic, still without clear consensus on the ideal location of mesh. The anatomic location of the mesh has an influence on how the mesh is incorporated with the tissues, the tensile strength of the repair and the abdominal wall, and finally the immune reaction between the mesh and the tissue ${ }^{[3]}$. We strive to summarize the advantages and limitations of these locations to make an argument for the ideal mesh plane for ventral hernia repair in abdominal wall reconstruction.

Onlay mesh placement is the placement of mesh on the anterior fascia and is sometimes referred to as a premuscular location of mesh $^{[4]}$. This technique, popularized by Chevrel in 1979, is typically approached in an open fashion with the placement of mesh over the anterior fascia following closure of the fascial defect. The key tenets of this approach include the reapproximation of the linea alba and fixation of mesh to the anterior fascia, which requires the creation of lipocutaneous flaps and the sacrifice of the periumbilical umbilical perforator vessels. The key to the onlay mesh is based on Chevrel's exploration of human cadavers anterior and posterior rectus sheaths. In his 1997 study on cadaveric specimen, Chevrel describes the burst strength of the anterior rectus sheath above the arcuate line to be the strongest portion of the abdominal wall which forms the basis for the onlay approach. He suggests that the strongest reinforcement for the abdominal wall is the combination of the native strength of the anterior rectus sheath in combination with the strength of polypropylene mesh ${ }^{[5]}$. 
The inlay (or interposition) technique, as defined by Parker et al. ${ }^{[6]}$, is characterized by an approach that places the mesh within the hernia/fascial defect with the mesh fixated to the edges of the neck of the hernia. By definition, the inlay placement of mesh requires a bridging mesh regardless of where the mesh is fixated. If the fascial defect closure is not achieved, this is also considered an interposition mesh. This approach can be completed open, as well as via a minimally invasive approach.

The sublay-retromuscular technique describes mesh placed posterior to the rectus muscle and anterior to the posterior rectus sheath. This plane continues below the arcuate line as the plane between the rectus abdominis muscle and the transversalis fascia ${ }^{[6]}$. This approach was originally described by Rives and Stoppa, and is characterized by opening the rectus sheath and defining the retrorectus plane posterior to the rectus abdominis muscle. The unique characteristics of this repair include the placement of mesh in the well vascularized retrorectus plane. The opening of this plane allows for the medialization and restoration of the linea alba, which results in offloading of tension on the suture line. The posterior fascia is approximated and the mesh is placed anteriorly in the plane between the rectus abdominis muscle and the posterior rectus fascia ${ }^{[3]}$. While this was previously exclusively an open approach as originally described, the sublay-retromuscular approach is now being increasingly performed via a minimally invasive approach. These approaches include combined endoscopic/open procedures as described by Schwarz et al. ${ }^{[7]}$ in the endoscopic mini/less open sublay repair. Additionally, this sublay-retromuscular approach has now been extensively described as the extended totally extraperitoneal repair, and can be performed both laparoscopically and robotically ${ }^{[8]}$. Belyansky et al. ${ }^{[9]}$ reported on this novel approach in 2018 and its advantages, including extraperitoneal suture closure of defects, wide mesh coverage in the sublay-retromuscular position with the use of minimal fixation, and an anecdotal appreciation for decreased pain associated with the repair. The sublay-preperitoneal technique describes mesh placement in the plane behind all of the abdominal wall muscles in front of the peritoneum. This technique is more often performed on the robotic platform, given its technical challenge in a laparoscopic approach ${ }^{[10]}$. The sublay-intraperitoneal technique describes mesh placement behind the abdominal wall muscles including the parietal peritoneum ${ }^{[11]}$. If done in an open fashion, the mesh is secured posteriorly to the posterior rectus sheath and the parietal peritoneum of the anterior abdominal. In a minimally invasive approach, both laparoscopically and robotically, the hernia sac is identified and its contents reduced. Although the fascial defect is more often closed in the robotic approach, the defect can also be closed in the laparoscopic approach $^{[12]}$. Regardless of defect closure, the mesh is then secured in place underlying fascia ${ }^{[13,14]}$.

\section{ADVANTAGES AND CURRENT DATA}

The topic of mesh selection has been an ongoing debate in the surgical community and will not serve as the focus of this review. However, regardless of mesh selection, the optimal location remains up for debate. Mesh implantation has been reported with both prosthetic and biologic varieties; however, multiple factors such as hospital contracts, surgeon experience, and cost drive the decision for mesh selection ${ }^{[13,14]}$. In Sosin et al.'s ${ }^{[15]} 2018$ meta-analysis of ventral hernia repairs, 6227 patients undergoing ventral hernia repair with mesh were aggregated in a total of 51 studies. The overall recurrence rate for all comers was $8.9 \%$ regardless of location of mesh. Notably, there was a statistically significant difference in recurrent rates that was dependent on the location of mesh. The lowest recurrence rate in this meta-analysis was in mesh placed in the sublay-retromuscular plane, with a $5.8 \%$ hernia recurrence rate $(P=0.023)$. Recurrence rates in the sublay-intraperitoneal and sublay-preperitoneal (summarily referenced as underlay in Sosin et al.'s ${ }^{[16]}$ metanalysis) mesh placement were $10.9 \%$ and $12.9 \%$, respectively. The highest hernia recurrence rate of $21.6 \%$ was observed in patients who underwent an inlay mesh placement ${ }^{[8,9]}$. Additionally, on repeated meta-analysis performed by Holihan et al. ${ }^{[16,17]}$, the sublay-retromuscular repair demonstrated a lower risk of recurrence and surgical site infection, when compared to onlay, inlay, and sublay-intraperitoneal or sublay-preperitoneal mesh approaches (range: OR: 0.45-0.79). The sublay-retromuscular repair was given a moderate recommendation of being the best approach when considering recurrence rate and surgical site 
infections ${ }^{[16]}$. When evaluating mesh location specific to synthetic versus biologic mesh placement, these distinctions did not remain. Specifically, in all patients who underwent synthetic mesh placement, mesh location was not a statistically significant predictor for recurrence rates $(P=0.95)^{[16]}$.

When evaluating overall complication rates, Sosin et al. $\mathrm{s}^{[15]}$ review highlights similar overall complication rates observed in ventral hernia repairs, which ranged from $32.6 \%$ to $39.1 \%$, regardless of mesh location with no statistically significant difference $(P=0.738)$. While the onlay approach is generally considered the least technically challenging approach to mesh placement, it has fallen out of favor due to the reported increased wound and mesh infection complications ${ }^{[18]}$ with approximately $7.6 \%$ of hernia repairs as of 2018 being performed in this plane. This is compared to greater than $65 \%$ of meshes being placed in the sublay-intraperitoneal, preperitoneal plane, or retromuscular plane in their pooled analysis of reported ventral hernia repairs ${ }^{[15]}$. The mean infection rate in the onlay subgroup was $14 \%$. The mean hematoma/ seroma complication rate was found to be $17.4 \%$, the highest amongst the four subgroups. However, the differences amongst complications in different mesh planes was not significant. The onlay approach's largest disadvantage is the mesh's direct contact with the environment during revision of the wound, which can lead to the subsequent wound complications observed in these studies ${ }^{[19]}$.

The inlay technique, which requires a bridging mesh, is performed when the fascial defect cannot be closed. Laparoscopic repair was the dominant approach for this mesh placement accounting for $72.6 \% v s$. $27.4 \%$ for open repairs. Infection rates in this approach was $12 \%$ and mean hematoma/seroma rate was $12.2 \%$, which did not significantly differ among the four techniques. Hernia recurrence was the highest in this subgroup, with a $21.6 \%$ hernia recurrence rate. The sublay-retromuscular approach to mesh placement can be achieved both via an open surgical approach or through minimally invasive techniques. The open approach remains the dominant surgical approach in Sosin et al.'s ${ }^{[15]}$ analysis, with $94 \%$ accounting for an open repair. The mean infection rate was $10.4 \%$ and mean hematoma/seroma rate was $11 \%$. This subgroup had the lowest rate of hernia recurrence, at only $5.8 \%(P=0.023)$. The closure of the rectus muscles over prosthetic mesh in a well vascularized plane has proven to result in decreased wound infection rates. The sublay-intraperitoneal technique was achieved both laparoscopically (63\%) and through an open surgical technique (37\%). The mean infection rate in this group was the highest in Sosin et al.'s ${ }^{[15]}$ analysis, at $17.7 \%$. This compares to only $10.2 \%$ in the sublay-retromuscular cohort; however, in this analysis, these were not found to be statistically significant. Mean hematoma/seroma rate was recorded as $11.5 \%$. Hernia recurrence in this group was $10.9 \%$, the second lowest rate based on anatomic mesh placement ${ }^{[15]}$. These data are summarized in Table 1 . These data corroborate previously reported outcomes by Holihan et al. ${ }^{[16]}$, who found the lowest odds of developing a surgical site infection in those with a sublay-retromuscular approach (OR: 0.449; 95\%CI: 0.12-1.16) when compared to onlay mesh placement. The sublay-intraperitoneal or sublay-preperitoneal was almost double the odds (OR: 0.878; 95\%CI: 0.29-1.99). Notably, infection rates are significantly different when evaluating open versus laparoscopic approach. This is demonstrated in Table 2. In Gokcal et al. s $^{[10]}$ single institution comparison of robotic preperitoneal and intraperitoneal ventral hernia repair, perioperative outcomes at three months were similar. Extremely short-term outcomes at three weeks demonstrated higher surgical site occurrences in the intraperitoneal cohort when compared to the preperitoneal cohort $(14 \%$ vs. $5.3 \%, P=0.042)$.

\section{MESH SELECTION}

Mesh selection is a multifaceted dilemma based on what is familiar to the surgeon, what is available to the surgeon based on institutional contracts and cost, and the approach to repair selected. However, at the core of selection are the properties of the mesh and these in general fall into two categories: biologic and synthetic. Similar to the lack of strong consensus on the optimal location for mesh placement, there remains lack of strong consensus on what type of mesh to use. While there is general consensus on the 
Table 1. Review of outcomes by mesh plane location ${ }^{[15]}$

\begin{tabular}{|c|c|c|c|c|c|}
\hline Outcomes & Onlay & Inlay & Sublay (Retromuscular) & Sublay (Intraperitoneal/Preperitoneal) & $P$ value \\
\hline Infection & $14.0 \%$ & $12.0 \%$ & $10.2 \%$ & $17.7 \%$ & 0.276 \\
\hline Seroma/Hematoma & $17.4 \%$ & $12.2 \%$ & $11.0 \%$ & $11.5 \%$ & 0.288 \\
\hline Mesh removal & $0.3 \%$ & $0.3 \%$ & $0.5 \%$ & $1.1 \%$ & 0.346 \\
\hline Recurrence & $12.9 \%$ & $21.6 \%$ & $5.8 \%$ & $10.9 \%$ & 0.023 \\
\hline Mortality & $0.3 \%$ & $0.3 \%$ & $0.5 \%$ & $0.5 \%$ & 0.929 \\
\hline Overall complication & $38.6 \%$ & $39.1 \%$ & $32.6 \%$ & $37.8 \%$ & 0.738 \\
\hline
\end{tabular}

Table 2. Review of outcomes by mesh plane location and surgical approach (open vs, laparoscopic) ${ }^{[15]}$

\begin{tabular}{lllccc}
\hline Outcomes & Onlay & Inlay & Sublay (Retromuscular) & Sublay (Intraperitoneal/Preperitoneal) & P value \\
\hline Open & & & & & \\
Seroma/Hematoma & $22.1 \%$ & $10.7 \%$ & $11.0 \%$ & $7.8 \%$ & $0.016^{*}$ \\
Infection & $9.6 \%$ & $20.9 \%$ & $12.1 \%$ & $17.8 \%$ & 0.121 \\
Recurrence & $9.9 \%$ & $25.4 \%$ & $6.7 \%$ & $10.9 \%$ & $0.020^{*}$ \\
Overall complication & $36.2 \%$ & $51.5 \%$ & $37.0 \%$ & $37.7 \%$ & 0.529 \\
Laparoscopic & & & & $3.5 \%$ & $0.044^{*}$ \\
Seroma/Hematoma & $\mathrm{N} / \mathrm{A}$ & $10.7 \%$ & $3.3 \%$ & $2.8 \%$ & 0.605 \\
Infection & $\mathrm{N} / \mathrm{A}$ & $1.3 \%$ & $0.1 \%$ & $4.2 \%$ & $0.041^{\star}$ \\
Recurrence & $\mathrm{N} / \mathrm{A}$ & $10.0 \%$ & $0.1 \%$ & $17.8 \%$ & 0.738 \\
Overall complication & $\mathrm{N} / \mathrm{A}$ & $24.1 \%$ & $6.2 \%$ & & \\
\hline
\end{tabular}

${ }^{\star}$ Denotes a statistically significant difference. N/A: Not applicable

use of a reinforcement material in the management of ventral/incision hernias, the composition of the reinforcement continues to be debated.

\section{Synthetic mesh}

Permanent synthetic mesh is the most commonly used reinforcement material in clinical practice. Sosin et al.' ${ }^{[15]}$ recent review of the literature demonstrated $68.5 \%$ of reviewed cases utilizing synthetic mesh, while only $31.5 \%$ of cases were performed with biologic mesh. Kingsnorth et al. ${ }^{[20]}$ reported as high as $90 \%$ of cases being performed with synthetic material. The breadth of synthetic mesh available on the market is vast and the products listed here, while not comprehensive, demonstrate the wide variety of products available [Table 3]. These meshes vary in their composition and can be further classified as permanent or bioabsorbable. The advantages of both types of synthetic mesh when compared to biologic mesh are their low cost. Although permanent synthetic mesh in general has the overall lowest cost and lowest recurrence rates, they are not recommended in grossly contaminated and infected fields and reportedly have higher rates of infection, discomfort, and adhesions encountered in re-operative fields. Synthetic meshes are marketed by several manufacturers and are usually made of polypropelene, expanded polytetrafluoroethylene, or polyethylene terephthalate polyester.

Bioabsorbable meshes were devised as an alternative to synthetic meshes offering a safer side effect profile in a contaminated field. They are made of the following materials and marketed under a variety of names by different manufacturers: polyglactin, polyglycolic acid, polyglycolic acid/trimethylene carbonate, poly4-hydroxybutyrate, and polyglycolide/polylactide/trimethylene carbonate. These degradable materials vary particularly in the time in which they degrade with products such as Polyglactin (Vicryl, Ethicon, USA) degrading in merely 1-3 months while, e.g., Phasix (Bard Davol Inc., USA) is a slowly resorbable mesh biosynthetic mesh with both the biocompatibility and resorbability of a biological mesh and the mechanical strength of a synthetic mesh. The drawback of these materials is the paucity of long-term data demonstrating efficacy with comparable recurrence rates. There are protocols in the pipeline looking at biosynthetics, such as Phasix (Bard Davol Inc., USA), and its long-term outcomes in Ventral Hernia Working Group (VHWG) Grade 3 wounds ${ }^{[21]}$. 
Table 3. Sample of synthetic mesh products by manufacture ${ }^{[24]}$

\begin{tabular}{ll}
\hline Synthetic mesh product name & \multicolumn{1}{c}{ Manufacture } \\
\hline Prolene & Boston Scientific \\
Parietex & Medtronic \\
Polutetrafluoroethylene & W. L. Gore \& Associates \\
Seri & Sofregen Medical Inc. \\
Marlex & CR Bard \\
Sepramesh & CR Bard \\
Proceed & Ethicon \\
ProGrip & Medtronic \\
Prolite & Atrium Medical Corp. \\
\hline
\end{tabular}

Table 4. Ventral hernia working group classification system for surgical site occurrence risk ${ }^{[22]}$

\begin{tabular}{ll}
\hline VHWG Grade & \multicolumn{1}{c}{ Characteristics } \\
\hline 1. Low risk & Low risk of complications \\
& No history of wound infections \\
2. Co-morbid & Smoker \\
& Obese \\
& Diabetic \\
& Immunosuppressed \\
& COPD \\
3. Potentially contaminated & Previous wound infection \\
& Stoma present \\
& Violation of the gastrointestinal tract \\
4. Infected & Infected mesh \\
& Septic dehiscence \\
\hline
\end{tabular}

VHWG: Ventral Hernia Working Group; COPD: chronic obstructive pulmonary disease

In 2010, the VHWG developed a grading system for surgeons to use to determine the complexity of the case with regards to risk of surgical site occurrence (SSO). At that time, this novel grading system created a framework by which a surgeon could assess the risk of SSO based on both the patient's comorbidities and the characteristics of the hernia to be repaired ${ }^{[22]}$. While this grading system does not take into account the size of the hernia defect or if the defect is the result of a recurrence, it created a uniform system by which to categorize wounds based on their SSO risk and provided recommendations as to which mesh types may be appropriate based on these categories of risk [Table 4$]^{[22]}$. With this grading system in mind, the VHWG recommends the use of a prosthetic reinforcement material in the case of all incisional/ventral hernias regardless of whether the midline fascia is reapproximated or not ${ }^{[22]}$. The working group concludes that synthetic mesh should be used in hernias without gross contamination, or Grade 1 categorized patients. In Sosin et al.'s ${ }^{[15]}$ review, there were notable differences in complications, infections, and the formation of seroma/hematoma in the placement of synthetic mesh in varying planes, as described in Table 5 . Recurrence rates and occurrence of mesh removal were statistically similar regardless of mesh plane.

\section{Biologic mesh}

Biologic mesh development occurred because of the need for a material that was believed to heal by tissue ingrowth as opposed to scar formation and encapsulation, which potentially would allow its utilization in an infected or contaminated field. Products on the market are in general created with a decellularized human, porcine, or bovine scaffold, whether dermis, pericardium, or intestinal mucosa. The extracellular collagen matrix is thought to encourage incorporation of the surrounding tissue by ingrowth of the fibrocollagenous tissue and blood vessels ${ }^{[23]}$. Table 6 lists the most commonly used biologic meshes currently on the market ${ }^{[24]}$. While many of the meshes perform in a similar manner, the unique qualities within these meshes include some chemical modifications to create cross-links in the collagen fibers, while others are xenogenic, and some are allogenic. Some have reported that the cross-linking nature of the meshes help to prevent degradation and increase the durability of the product and the repair; however, they 
Table 5. Review of outcomes by mesh plane location with the use of synthetic mesh ${ }^{[15]}$

\begin{tabular}{lllccc}
\hline Synthetic mesh & Onlay & Inlay & Sublay (Retromuscular) & Sublay (Intraperitoneal/Preperitoneal) & $\boldsymbol{P}$ value \\
\hline Infection & $7.6 \%$ & $1.3 \%$ & $10.3 \%$ & $2.6 \%$ & 0.008 \\
Seroma/Hematoma & $19.7 \%$ & $10.8 \%$ & $9.5 \%$ & $3.5 \%$ & 0.003 \\
Mesh removal & $0.0 \%$ & $0.3 \%$ & $0.8 \%$ & $0.5 \%$ & 0.594 \\
Recurrence & $4.7 \%$ & $5.7 \%$ & $5.2 \%$ & $4.4 \%$ & 0.952 \\
Overall complication & $31.8 \%$ & $19.1 \%$ & $31.3 \%$ & $16.6 \%$ & 0.022 \\
\hline
\end{tabular}

Table 6. Biologic mesh types ${ }^{[24]}$

\begin{tabular}{ll}
\hline Biologic mesh types & \multicolumn{1}{c}{ Component } \\
\hline Alloderm & Human dermis \\
Allomax & Human dermis \\
Collamend & Porcine dermis \\
Fortagen & Porcine intestine collagen \\
Peramcol & Cross-linked porcine dermis collagen \\
Periguard & Bovine pericardium \\
Strattice & Non-cross-linked porcine dermis collagen \\
SurgiMend & Bovine dermis \\
Surgisis & Porcine intestine collagen \\
Tutopas & Bovine pericardium \\
Veritas & Bovine pericardium \\
XenMatrix & Porcine dermis \\
\hline
\end{tabular}

can cause a heightened foreign body reaction and early inflammatory response ${ }^{[24]}$. Others have reported that the crosslinking can contribute to the lack of integration of the mesh with the surrounding tissue with resultant encapsulation of the mesh and may result in decreased tensile strength ${ }^{[25]}$. Non-crosslinked products, such as Strattice (Medtronic Inc., Dublin, Ireland), are reported to demonstrate fewer adhesions and complications, when compared to the cross-linked products, such as Permacol (Medtronic Inc., Dublin, Ireland). The human acellular dermal matrices, such as Alloderm (Allergan Plc, Dublin, Ireland), have been shown to have higher failure rates including eventration secondary to higher elastin content ${ }^{[26]}$.

While there are no randomized control trials comparing synthetic versus biologic mesh in VHWG Grade $>1$, the VHWG recommends the use of biologic mesh in incisional hernias with VHWG Grade 4, which describes a wound that was involved with infected mesh or a septic dehiscence. There are ample studies suggesting the increased rate of reoperation and need for removal of mesh due to additional mesh infections when placing synthetic mesh in a grossly contaminated or infected field. In the setting of a potentially contaminated field, or VHWG 3, the VHWG advises against the use of synthetic mesh and acknowledges there may be benefit to the use of a biologic prosthesis. In general, no strong recommendations exist for the absolute use of specific biologic prosthesis; however, Liang et al. ${ }^{[27]}$, reiterated the need for randomized control trials comparing synthetic, biologic, and bioabsorbable meshes to provide clarity on their respective uses ${ }^{[2]}$. Nonetheless, many experts avoid the routine use of biologic mesh in clean cases and reserve its use in the setting of high risk patients and grossly contaminated cases ${ }^{[27]}$. In our practice, we limit its use to the grossly contaminated field. Sosin et al.'s ${ }^{[15]}$ review demonstrates overall unfavorable outcomes occurred when compared to synthetic meshes. Plane selection with the use of biologic mesh were similar in overall complications, except in the occurrence of hematomas/seromas in which the underlay location of mesh resulted in the lowest occurrence of hematomas/seromas [Table 7]. However, when looking at the occurrence of overall infections in the synthetic mesh group compared to that of the biologic mesh group, plane for plane, there is an overwhelmingly higher occurrence of wound infection in the biologic cohort when compared to the synthetic cohort. This may be explained by the general use of synthetic mesh in a clean operative field compared to that of biologic mesh. Nonetheless, even in the preferred sublay-intraperitoneal plane, there was as high as $19.2 \%$ infection occurrence with the use of biologic mesh compared to only $2.6 \%$ in the synthetic mesh cohort. 
Table 7. Review of outcomes by mesh plane location with the use of biologic mesh ${ }^{[15]}$

\begin{tabular}{|c|c|c|c|c|c|}
\hline Biologic mesh & Onlay & Inlay & Sublay (Retromuscular) & Sublay (Intraperitoneal/Preperitoneal) & $P$ value \\
\hline Infection & $21.3 \%$ & $23.3 \%$ & $18.1 \%$ & $19.2 \%$ & 0.814 \\
\hline Seroma/Hematoma & $32.3 \%$ & $10.8 \%$ & $11.2 \%$ & $8.0 \%$ & $<0.001$ \\
\hline Mesh removal & $0.0 \%$ & $0.0 \%$ & $1.6 \%$ & $0.5 \%$ & 0.359 \\
\hline Recurrence & $28.6 \%$ & $29.1 \%$ & $11.6 \%$ & $11.2 \%$ & 0.016 \\
\hline Overall complication & $57.3 \%$ & $54.2 \%$ & $40.9 \%$ & $40.9 \%$ & 0.197 \\
\hline
\end{tabular}

\section{CONCLUSION}

Half a million patients undergo ventral hernia repair annually in the United States as a result of incisional hernias, failed repairs, de novo abdominal wall defects, and abdominal catastrophes. Although the standard approach of hernia repair has been well studied, the ideal anatomic location of mesh placement is still highly debated. Sosin et al.'s ${ }^{[15]}$ systematic review of mesh placement found that anatomic location can change outcomes in hernia recurrence. Analysis of 51 articles showed that, of the four mesh techniques, namely onlay, interposition, sublay-retromuscular, and sublay-preperitoneal/sublay-peritoneal, the sublayretromuscular approach is associated with the lowest recurrence rate, whereas the interposition technique is associated with the highest recurrence rate. There was no statistical difference in other complication rates among the four groups, which included postoperative infection, hematoma/seroma formation, mesh explantation, and mortality. Overwhelmingly, the inlay placement of mesh is the least favored and should be avoided if possible. In the minimally invasive approach, both robotically and laparoscopically, the sublay-preperitoneal/sublay-intraperitoneal repair has proven very useful with similar perioperative complications and recurrence rates. In regards to mesh selection, in accordance with the recommendations of the VHWG, we recommend that all ventral hernias be reinforced with mesh regardless of whether the midline fascia can be reapproximated ${ }^{[2]}$. While strong recommendations for the use of synthetic versus biologic mesh are unclear in patients with VHWG Grades 2 and 3, biologic mesh's benefit is clear in grossly contaminated wounds and synthetic mesh is recommended in VHWG Grade 1 patients.

\section{DECLARATIONS}

\section{Authors' contributions}

Conceived the concept of the review and are the primary authors of the manuscript: Alimi Y, Bhanot P

Performed significant writing and review of the final manuscript: Merle $\mathrm{C}$

Provided feedback, essential data gathering and review of the manuscript: Sosin $\mathrm{M}$

Provided principal figures in the article: Mahan M

All authors read and approved the final manuscript.

\section{Availability of data and materials}

Not applicable.

\section{Financial support and sponsorship}

None.

\section{Conflicts of interest}

All authors declared no conflicts of interest. Dr. Parag Bhanot is a consultant to Allergan.

\section{Ethical approval and consent to participate}

Not applicable.

\section{Consent for publication}

Not applicable. 


\section{Copyright}

(C) The Author(s) 2020.

\section{REFERENCES}

1. Bedewi MA, El-Sharkawy MS, Al Boukai AA, Al-Nakshabandi N. Prevalence of adult paraumbilical hernia. Assessment by highresolution sonography: a hospital-based study. Hernia 2012;16:59-62.

2. Colavita PD, Walters AL, Tsirline VB, Belyansky I, Lincourt AE, et al. The regionalization of ventral hernia repair: occurrence and outcomes over a decade. Am Surg 2013;79:693-701.

3. Timmermans L, de Goede B, van Dijk SM, Kleinrensink GJ, Jeekel J, et al. Meta-analysis of sublay versus onlay mesh repair in incisional hernia surgery. Am J Surg 2014;207:980-8.

4. Haskins IN, Voeller GR, Stoikes NF, Webb DL, Chandler RG, et al. Onlay with adhesive use compared with sublay mesh placement in ventral hernia repair: was chevrel right? An Americas hernia society quality collaborative analysis. J Am Coll Surg 2017;224:962-70.

5. Rath AM, Zhang J, Chevrel JP. The sheath of the rectus abdominis muscle: an anatomical and biomechanical study. Hernia 1997;1:139-42.

6. Parker SG, Wood CPJ, Sanders DL, Windsor ACJ. Nomenclature in abdominal wall hernias: is it time for consensus? World J Surg 2017;41:2488-91.

7. Schwarz J, Reinpold W, Bittner R. Endoscopic mini/less open sublay technique (EMILOS)-a new technique for ventral hernia repair. Langenbecks Arch Surg 2017;402:173-80.

8. Cox TC, Pearl JP, Ritter EM. Rives-Stoppa incisional hernia repair combined with laparoscopic separation of abdominal wall components: a novel approach to complex abdominal wall closure. Hernia 2010;14:561-7.

9. Belyansky I, Daes J, Radu VG, Balasubramanian R, Zahiri HR, et al. A novel approach using the enhanced-view totally extraperitoneal (eTEP) technique for laparoscopic retromuscular hernia repair. Surg Endosc 2018;32:1525-32.

10. Gokcal F, Morrison S, Kudsi OY. Short-term comparison between preperitoneal and intraperitoneal onlay mesh placement in robotic ventral hernia repair. Hernia 2019;23:957-67.

11. Muysoms F, Campanelli G, Champault GG, DeBeaux AC, Dietz UA, et al. EuraHS: the development of an international online platform for registration and outcome measurement of ventral abdominal wall hernia repair. Hernia 2012;16:239-50.

12. Chelala E, Baraké H, Estievenart J, Dessily M, Charara F, et al. Long-term outcomes of 1326 laparoscopic incisional and ventral hernia repair with the routine suturing concept: a single institution experience. Hernia 2016;20:101-10.

13. Nobaek S, Rogmark P, Petersson U, Hu SB, Sun P, et al. Incisional hernia: complications \& quality of life. Hernia 2015;19 (Suppl 1):S51-6.

14. Criss CN, Petro CC, Krpata DM, Seafler CM, Lai N, et al. Functional abdominal wall reconstruction improves core physiology and quality-of-life. Surgery 2014;156:176-82.

15. Sosin M, Nahabedian MY, Bhanot P. The perfect plane: a systematic review of mesh location and outcomes, update 2018. Plast Reconstr Surg 2018;142:S107-16.

16. Holihan JL, Hannon C, Goodenough C, Goodenough C, Flores-Gonzalez JR, et al. Ventral hernia repair: a meta-analysis of randomized controlled trials. Surg Infect 2017;18:647-58.

17. Holihan JL, Nguyen DH, Nguyen MT, Mo J, Kao LS, et al. Mesh location in open ventral hernia repair: a systematic review and network meta-analysis. World J Surg 2016;40:89-99.

18. Köckerling F, Lammers B. Open intraperitoneal onlay mesh (IPOM) technique for incisional hernia repair. Front Surg 2018;5:66.

19. Korenkov M, Paul A, Sauerland S, Campanelli G, Champault GG, et al. Classification and surgical treatment of incisional hernia. Langenbecks Arch Surg 2001;386:65-73.

20. Sanders D, Kingsnorth A. The modern management of incisional hernias. BMJ 2012;344:e2843.

21. van Rooijen MMJ, Jairam AP, Tollens T, Jørgensen LN, de Vries Reilingh TS, et al. A post-market, prospective, multi-center, single-arm clinical investigation of PhasixTM mesh for VHWG grade 3 midline incisional hernia repair: a research protocol. BMC Surg 2018;18:104.

22. Breuing K, Butler CE, Ferzoco S, Franz M, Hultman CS, et al. Incisional ventral hernias: review of the literature and recommendations regarding the grading and technique of repair. Surgery 2010;148:544-58.

23. Slater NJ, van der Kolk M, Hendriks T, van Goor H, Bleichrodt RP. Biologic grafts for ventral hernia repair: a systematic review. Am J Surg 2013;205:220-30.

24. Rastegarpour A, Cheung M, Vardhan M, Ibrahim MM, Facs CEB, et al. Surgical mesh for ventral incisional hernia repairs: understanding mesh design. Plast Surg (Oakv) 2016;24:41-50.

25. Jin J, Rosen MJ, Blatnik J, McGee MF, Williams CP, et al. Use of acellular dermal matrix for complicated ventral hernia repair: does technique affect outcomes? J Am Coll Surg 2007;205:654-60.

26. Huntington CR, Cox TC, Blair LJ, Schell S, Randolph D, et al. Biologic mesh in ventral hernia repair: outcomes, recurrence, and charge analysis. Surgery 2016;160:1517-27.

27. Liang MK, Holihan JL, Itani K, Alawadi ZM, Flores Gonzalez JR, et al. Ventral hernia management: expert consensus guided by systematic review. Ann Surg 2017;265:80-9. 